\title{
A FAST ALGORITHM FOR MATRIX BALANCING
}

\author{
PHILIP A. KNIGHT* AND DANIEL RUIZ ${ }^{\dagger}$
}

\begin{abstract}
As long as a square nonnegative matrix $A$ contains sufficient nonzero elements, then the matrix can be balanced, that is we can find a diagonal scaling of $A$ that is doubly stochastic. A number of algorithms have been proposed to achieve the balancing, the most well known of these being the Sinkhorn-Knopp algorithm. In this paper we derive new algorithms based on inner-outer iteration schemes. We show that the Sinkhorn-Knopp algorithm belongs to this family, but other members can converge much more quickly. In particular, we show that while stationary iterative methods offer little or no improvement in many cases, a scheme using a preconditioned conjugate gradient method as the inner iteration can give quadratic convergence at low cost.
\end{abstract}

Key words. Matrix balancing, Sinkhorn-Knopp algorithm, doubly stochastic matrix, conjugate gradient iteration.

AMS subject classifications. 15A48, 15A51, 65F10, 65H10.

1. Introduction. For at least 70 years, scientists in a wide variety of disciplines have attempted to transform square nonnegative matrices into doubly stochastic form by applying diagonal scalings. That is, given $A \in \mathbb{R}^{n \times n}, A \geq 0$, find diagonal matrices $D_{1}$ and $D_{2}$ so that $P=D_{1} A D_{2}$ is doubly stochastic. Motivation for achieving this balance include interpreting economic data [2], preconditioning sparse matrices [13], understanding traffic circulation [11] and ordering nodes in a graph [9]. In all of these applications, one of the main methods considered is the Sinkhorn-Knopp algorithm ${ }^{1}$. This is an iterative process that attempts to find $D_{1}$ and $D_{2}$ by alternately normalising columns and rows in a sequence of matrices starting with $A$. Convergence conditions for this algorithm are well known: if $A$ has full support ${ }^{2}$ then the algorithm will converge linearly with asymptotic rate equal to the square of the subdominant singular value of $P[18,19,9]$.

Clearly, in some cases the convergence will be painfully slow. The principal aim of this paper is to derive some new algorithms for the matrix balancing problem with an eye on speed, especially for large systems. First we look at a simple Newton method for symmetric matrices, closely related to a method proposed by Khachiyan and Kalantari [8] for positive definite (but not necessarily nonnegative) matrices. We

${ }^{*}$ Department of Mathematics, University of Strathclyde, 26 Richmond Street, Glasgow G1 1XH Scotland (pk@maths.strath.ac.uk). This work was supported in part by a grant from the Carnegie Trust for the Universities of Scotland.

${ }^{\dagger}$ ENSEEIHT 2, rue Charles Camichel, Toulouse, France (Daniel.Ruiz@enseeiht.fr)

${ }^{1}$ The algorithm has been given many different names, Sinkhorn-Knopp is the name usually adopted by the linear algebra community

${ }^{2}$ Full support is defined in $\S 3$.

Dagstuhl Seminar Proceedings 07071

Web Information Retrieval and Linear Algebra Algorithms http://drops.dagstuhl.de/opus/volltexte/2007/1073 
will show that as long as Newton's method produces a sequence of positive iterates, the Jacobians we generate will be positive semi-definite.

To apply Newton's method exactly we require a linear system solve at each step, and this is usually prohibitively expensive. We therefore look at iterative techniques for approximating the solution at each step. First we look at splitting methods and we see that the Sinkhorn-Knopp algorithm is a member of this family of methods. We give an asymptotic bound on the (linear) rate of convergence of these methods. Next we look at a preconditioned conjugate gradient method for solving the inner iteration. We discuss implementation details and show that asymptotically, superlinear convergence is achievable. By implementing an Armijo-type rule we ensure that our iterates retain positivity and we demonstrate the reliability and speed of our method in tests.

A number of authors have presented alternative techniques for balancing matrices that can converge faster than the Sinkhorn-Knopp algorithm. For example, Parlett and Landis [16] look at some simple ways of trying to accelerate the convergence by focusing on reducing statistics such as the standard deviation between row sums of the iterates. In certain cases, they show great improvement is possible, suggesting that the rate on convergence is not dependent on the singular values of $P$; but they also give examples where their alternatives perform significantly worse. We include a comparison of one of these algorithms against our proposed approach in $\S 6$. Linial et al. [12] use a similar approach to [16] in the context of estimating matrix permanents, although their upper bound on iteration counts $\left(O\left(n^{7}\right)\right)$ makes them distinctly unappealing for large problems!

A completely different approach is to view matrix balancing as an optimisation problem. There are many alternative formulations, perhaps the first being that of Marshall and Olkhin [14] who show that balancing is equivalent to minimizing the bilinear form $x^{T} A y$ subject to the constraints $\Pi x_{i}=\Pi y_{i}=1$. However, the experimental results we have seen for optimisation techniques for balancing $[14,17,3]$ suggest that these methods are not particularly cheap to implement.

2. Newton's method. Let $\mathcal{D}: \mathbb{R}^{n} \rightarrow \mathbb{R}^{n \times n}$ represent the operator that turns a vector into a diagonal matrix, $\mathcal{D}(x)=\operatorname{diag}(x)$, and let $e$ represent a vector of ones. Then to balance a nonnegative matrix, $A$, we need to find positive vectors $r$ and $c$ such that

$$
\mathcal{D}(r) A \mathcal{D}(c) e=\mathcal{D}(r) A c=e, \mathcal{D}(c) A^{T} r=e .
$$

Rearranging these identities gives

$$
c=\mathcal{D}\left(A^{T} r\right)^{-1} e, \quad r=\mathcal{D}(A c)^{-1} e,
$$


and one way of writing the Sinkhorn-Knopp algorithm [6, 9] is as

$$
c_{k+1}=\mathcal{D}\left(A^{T} r_{k}\right)^{-1} e, \quad r_{k+1}=\mathcal{D}\left(A c_{k+1}\right)^{-1} e .
$$

If $A$ is symmetric then (2.1) can be simplified. To achieve balancing we need a vector $x$ that satisfies

$$
f(x)=\mathcal{D}(x) A x-e=0 .
$$

This leads to the iterative step

$$
x_{k+1}=\mathcal{D}\left(A x_{k}\right)^{-1} e .
$$

Exactly the same calculations are performed as for (2.2), one simply extracts $r_{k}$ and $c_{k}$ from alternate iterates [9]. If $A$ is nonsymmetric, one can use (2.4) on

$$
S=\left[\begin{array}{cc}
0 & A \\
A^{T} & 0
\end{array}\right] .
$$

An obvious alternative to the Sinkhorn-Knopp algorithm is to solve (2.3) with Newton's method. Differentiating $f(x)$ gives

$$
J(x)=\frac{\partial}{\partial x}(\mathcal{D}(x) A x-e)=\mathcal{D}(x) A+\mathcal{D}(A x),
$$

a result that is easily confirmed by componentwise calculation or with tensor algebra, hence Newton's method can be written

$$
x_{k+1}=x_{k}-\left(\mathcal{D}\left(x_{k}\right) A+\mathcal{D}\left(A x_{k}\right)\right)^{-1}\left(\mathcal{D}\left(x_{k}\right) A x_{k}-e\right) .
$$

We can rearrange this equation to get

$$
\left(\mathcal{D}\left(x_{k}\right) A+\mathcal{D}\left(A x_{k}\right)\right) x_{k+1}=\mathcal{D}\left(A x_{k}\right) x_{k}+e,
$$

so,

$$
\begin{aligned}
\left(A+\mathcal{D}\left(x_{k}\right)^{-1} \mathcal{D}\left(A x_{k}\right)\right) x_{k+1} & =\mathcal{D}\left(x_{k}\right)^{-1}\left(\mathcal{D}\left(A x_{k}\right) x_{k}+e\right) \\
& =A x_{k}+\mathcal{D}\left(x_{k}\right)^{-1} e
\end{aligned}
$$

and we can set up each Newton iteration by performing some simple vector operations and updating the diagonal on the left hand side. This matrix plays a key role in our analysis and we introduce the notation

$$
\mathcal{A}_{k}=A+\mathcal{D}\left(x_{k}\right)^{-1} \mathcal{D}\left(A x_{k}\right) .
$$

Note that the matrix on the lefthand side of (2.5) inherits the symmetry of $A$. This algorithm can be implemented by applying one's linear solver of choice. In practical 
applications, it makes sense to apply an inner-outer iteration scheme. In $\S 4$ and $\S 5$ we look at some efficient ways of doing this. In particular, we look at how to deal with the nonsymmetric case. Here, we can use the same trick we used to derive (2.4) but, as we'll see, the resulting linear systems are singular.

The idea of using Newton's method to solve the scaling problem is not new and was first proposed by Khachiyan and Kalantari in [8]. Instead of (2.3), the equivalent equation

$$
A x-\mathcal{D}(x)^{-1} e=0
$$

is considered. The authors were interested in the problem of scaling symmetric positive definite (SPD) matrices, rather than nonnegative matrices. In this case, the scaled matrix need not be doubly stochastic. While its row and column sums are 1, it may contain negative entries. The authors did not consider the practical application of their algorithm, although the methods we look at in this paper can be adapted to work for their formulation. In $\S 5$ we explain why our approach leads to faster convergence.

Newton's method is also used as a method for solving the balancing problem for symmetric matrices in [13]. Here the authors work with

$$
\left(I-\frac{e e^{T}}{n}\right) \mathcal{D}(A x) x=0
$$

instead of (2.3). They then use a Gauss-Seidel Newton method to solve the problem and show that this approach can give significant improvements over the SinkhornKnopp algorithm. We will develop this idea in $\S 4$.

Yet another formulation of (2.4) can be found in [5], where the author suggests the resulting equation is solved by Newton's method. However no attempt is made to implement the suggested algorithm and the fact that only the righthand side changes in the linear system that is solved at each step suggests that rapid convergence is unlikely in general.

3. Properties of $\mathcal{A}_{k}$. In order that we can solve the balancing problem efficiently, in particular when $A$ is large and sparse, we will use iterative methods to approximately solve the linear system in (2.5). There are a number of possibilities to choose as $\mathcal{A}_{k}$, the matrix on the lefthand side of the expression, is symmetric positive semidefinite. This is a consequence of the following result (which doesn't require symmetry).

THEOREM 3.1. Suppose that $A \in \mathbb{R}^{n \times n}$ is nonnnegative and $y \in \mathbb{R}^{n}$ is positive. Let $D=\mathcal{D}(A y) \mathcal{D}(y)^{-1}$. Then for all $\lambda \in \sigma(A+D), \operatorname{Re}(\lambda) \geq 0$.

Proof. Note that $A+D$ is similar to

$$
\mathcal{D}(y)^{-1}(A+D) \mathcal{D}(y)=\mathcal{D}(y)^{-1} A \mathcal{D}(y)+\mathcal{D}(y)^{-1} \mathcal{D}(A y)
$$




$$
\begin{aligned}
& =\mathcal{D}(y)^{-1} A \mathcal{D}(y)+\mathcal{D}\left(\mathcal{D}(y)^{-1} A \mathcal{D}(y) e\right) \\
& =B+\mathcal{D}(B e)
\end{aligned}
$$

where $B=\mathcal{D}(y)^{-1} A \mathcal{D}(y)$. Now $B e$ is simply the vector of row sums of $B$ and so adding this to the diagonal of $B$ gives us a diagonally dominant matrix. Since the diagonal is nonnegative, the result follows from Gershgorin's theorem.

If $A$ has a nonzero entry on its diagonal then at least one of the rows of $B+\mathcal{D}(B e)$ will be strongly diagonally dominant and, by a theorem of Taussky [20], it will be nonsingular. We can also ensure that $\mathcal{A}_{k}$ is nonsingular by imposing conditions on the connectivity between the nonzeros in $A$. We can establish the following result.

THEOREM 3.2. Suppose that $A \in \mathbb{R}^{n \times n}$ is nonnnegative. If $A$ is fully indecomposable then $\mathcal{A}_{k}$ is nonsingular.

Recall that a $A$ is fully indecomposable if it is impossible to find permutation matrices $P$ and $Q$ such that

$$
P A Q=\left[\begin{array}{cc}
A_{1} & 0 \\
A_{2} & A_{3}
\end{array}\right]
$$

with $A_{1}$ and $A_{2}$ square (a generalisation of irreducibility).

The proof of Theorem 3.2 is postponed to the end of the section. However in many cases we will not be able to satisfy its conditions. For example, if $A$ is nonsymmetric and we use Newton's method to balance

$$
S=\left[\begin{array}{cc}
0 & A \\
A^{T} & 0
\end{array}\right],
$$

then $S$ is not fully indecomposable. In fact it is straightforward to show that in this case the linear systems we have to solve are singular.

We'd also like to use our new algorithms on any nonnegative matrix for which balancing is possible, namely any matrix which has total support $(A \geq 0$ has total support if $A \neq 0$ and all the nonzero elements lie on a positive diagonal). Matrices that have total support but that aren't fully indecomposable also lead to singular systems in (2.5).

Singularity in these cases is not problematic as the systems are consistent. In fact, as Theorem 3.5 shows, we can go further, and we can solve the systems whenever $A$ has support ( $A \geq 0$ has support if it has a positive diagonal). We will investigate what happens when we use our algorithms on matrices without total support in $\S 6$.

We need some preliminary results.

LEMMA 3.3. Suppose that $A \in \mathbb{R}^{n \times n}$ is a symmetric nonnegative matrix with support. Then there is a permutation P such that PAP is a block diagonal matrix in which all the diagonal blocks are irreducible. 
Proof. We show this by induction on $n$. Clearly it is true if $n=1$. Suppose $n>1$ and our hypothesis is true for all matrices of dimension smaller than $n$. If $A$ is irreducible there is nothing to prove, otherwise we can find a permutation $Q$ such that

$$
Q A Q=\left[\begin{array}{cc}
A_{1} & C \\
0 & A_{2}
\end{array}\right]=\left[\begin{array}{cc}
A_{1} & 0 \\
0 & A_{2}
\end{array}\right],
$$

where $C=0$ by symmetry. Since $A$ has support it follows that, $Q A Q$ has a positive diagonal, hence $A_{1}$ and $A_{2}$ must each have support, too and we can apply our inductive hypothesis.

Lemma 3.4. Suppose that $A \geq 0$ has support and let $B=A+\mathcal{D}(A e)$. If either $A$ is symmetric or it is irreducible, the null space of $B$ is orthogonal to $e$.

Proof. If $B$ is nonsingular we have nothing to prove, so we assume it is singular (and hence by Taussky's theorem, $A$ has an empty main diagonal).

Suppose now that $B$ is irreducible. It follows from the Perron-Frobenius theorem that if $B$ is irreducible then the null space is one dimensional and, since it is weakly diagonally dominant, all components are of equal modulus (for a proof, see Theorem 2.3 and Remark 2.9 in [10]). All that remains is to show that there are an equal number of positive and negative components in elements from the null space.

We choose a permutation $i_{1}, i_{2}, \ldots, i_{n}$ of $1, \ldots, n$ such that for $1 \leq j \leq n, b_{i_{j} i_{j+1}} \neq$ 0 , where $i_{n+1}=i_{1}$. Such a permutation exists because $A$ has support and has an empty main diagonal. Now suppose that $x$ is in the null space of $B$. Since $B$ is diagonally dominant, the sign of $x_{i}$ must be opposite to that of $x_{j}$ whenever $b_{i j} \neq 0$ $(i \neq j)$. By construction, $x_{i_{j}}=-x_{i_{j+1}}$ for $1 \leq j \leq n$. This is only possible if $n$ is even, in which case $x^{T} e=0$, as required.

If $B$ is not irreducible but is symmetric then (by the previous lemma) $P B P=$ $\operatorname{diag}\left(B_{1}, B_{2}, \ldots, B_{k}\right)$ where the $B_{i}$ are irreducible. Since the null space of $B$ is formed from a direct sum of the null space of the diagonal blocks, it too must be orthogonal to $e$.

THEOREM 3.5. Suppose that $A \in \mathbb{R}^{n \times n}$ is a symmetric nonnegative matrix with support and that $y>0$. The system

$$
\left(A+\mathcal{D}(A y) \mathcal{D}(y)^{-1}\right) z=A y+\mathcal{D}(y)^{-1} e
$$

is consistent.

Proof. Let $A_{y}=\mathcal{D}(y) A \mathcal{D}(y)$. Since $\left(A_{y}+\mathcal{D}\left(A_{y} e\right)\right)$ is symmetric, elements orthogonal to its null space must lie in its range, so as a consequence of the previous lemma, we can find a vector $c$ such that

$$
\left(A_{y}+\mathcal{D}\left(A_{y} e\right)\right) c=e
$$


Let $z=y / 2+\mathcal{D}(y) c$. Then

$$
\begin{aligned}
\left(A+\mathcal{D}(y)^{-1} \mathcal{D}(A y)\right) z= & \frac{1}{2}\left(A+\mathcal{D}(A y) \mathcal{D}(y)^{-1}\right) y \\
& +\mathcal{D}(y)^{-1}(\mathcal{D}(y) A \mathcal{D}(y) c+\mathcal{D}(A y) \mathcal{D}(y) c) \\
= & A y+\mathcal{D}(y)^{-1}\left(A_{y}+\mathcal{D}\left(A_{y} e\right) c\right. \\
= & A y+\mathcal{D}(y)^{-1} e .
\end{aligned}
$$

While we have proved that Newton's step will converge if $x_{k}>0$, we have not shown that $x_{k+1}>0$. In fact, it needn't be, and this will be an important consideration for us in developing balancing methods in the later sections.

To finish the section, we prove Theorem 3.2.

Proof. Let $B=\mathcal{D}\left(x_{k}\right)^{-1} A \mathcal{D}\left(x_{k}\right)$ and consider $B+\mathcal{D}(B e)$. Suppose that this matrix is singular and $v$ lies in its null space. We can apply Lemma 3.4, and we know that exactly half of its components are positive and half are negative. Suppose that $P$ permutes $v$ so that the first half of the entries of $P v$ are positive. Then

$$
P(B+\mathcal{D}(B e)) P=\left[\begin{array}{cc}
D_{1} & B_{1} \\
B_{2} & D_{2}
\end{array}\right],
$$

where $D_{1}$ and $D_{2}$ are diagonal: otherwise, diagonal dominance would force some of the entries of $(B+\mathcal{D}(B e)) v$ to be nonzero. Hence

$$
B=\left[\begin{array}{cc}
0 & B_{1} \\
B_{2} & 0
\end{array}\right] .
$$

But such a matrix is not fully indecomposable, contradicting our hypothesis.

4. Stationary iterative methods. If we only want to solve (2.5) approximately, the simplest class of methods to consider is that of stationary iterative methods, in particular splitting methods. As $\mathcal{A}_{k}$ is symmetric positive semi-definite we know that many of the common splitting methods will converge.

This approach is used in [13] to solve the formulation of the balancing problem given in (2.7). Here, the authors use Gauss-Seidel to solve the Newton step and demonstrate that the rate of convergence is frequently faster than that of the SK algorithm. Suppose that the symmetric matrix $A$ can be balanced so that $P=D A D$ is doubly stochastic. Following the standard analysis for splitting methods, they give a bound on the rate of convergence in the neighbourhood of the solution, relating it to the modulus of the second largest eigenvalue of $L^{-1} U$, where $L$ is the lower triangular part and $U$ the strictly upper-triangular part of $P+I-2 e e^{T} / n .^{3}$

\footnotetext{
${ }^{3}$ The bound is not in terms of $\rho\left(L^{-1} U\right)$ as the matrix in the lefthand side of (2.7) is singular.
} 
In this section we extend the results in [13]. We use (2.5) rather than (2.7) as it leads to particularly simple representations of the methods.

Suppose that $\mathcal{A}_{k}=M-N$ where $M$ is nonsingular. A splitting method for (2.5) can then be written

$$
\begin{aligned}
M x_{k+1} & =N x_{k}+A x_{k}+\mathcal{D}\left(x_{k}\right)^{-1} e \\
& =\left(M-\mathcal{D}\left(x_{k}\right)^{-1} \mathcal{D}\left(A x_{k}\right)\right) x_{k}+\mathcal{D}\left(x_{k}\right)^{-1} e \\
& =M x_{k}-\mathcal{D}\left(A x_{k}\right) e+\mathcal{D}\left(x_{k}\right)^{-1} e \\
& =M x_{k}-A x_{k}+\mathcal{D}\left(x_{k}\right)^{-1} e .
\end{aligned}
$$

In other words, our iteration can be written

$$
x_{k+1}=x_{k}+M^{-1}\left(\mathcal{D}\left(x_{k}\right)^{-1} e-A x_{k}\right) .
$$

By fixing $M$ for a number of steps, we get an inner-outer iteration for solving the balancing problem. Strictly speaking, the inner iteration is not stationary as $\mathcal{D}\left(x_{k}\right)^{-1} e-$ $A x_{k}$ is updated at every step. However, we can update $M$ at each step at minimal cost-we only need to update its diagonal and the vectors we need for this are available to us. So for optimum performance we recommend performing the update at every step.

Note that if we choose $M=\mathcal{D}\left(x_{k}\right)^{-1} \mathcal{D}\left(A x_{k}\right)$ in (4.1), then the resulting iteration is

$$
\begin{aligned}
x_{k+1} & =x_{k}+\mathcal{D}\left(x_{k}\right) \mathcal{D}\left(A x_{k}\right)^{-1}\left(\mathcal{D}\left(x_{k}\right)^{-1} e-A x_{k}\right) \\
& =x_{k}+\mathcal{D}\left(A x_{k}\right)^{-1} e-\mathcal{D}\left(x_{k}\right) e \\
& =\mathcal{D}\left(A x_{k}\right)^{-1} e
\end{aligned}
$$

and we recover (2.4).

We can use an approach similar to that in [13] to bound the rate of convergence for many standard splitting methods applied to (4.1). For example, the Jacobi method and successive over relaxation are covered by the following result, where $X \circ Y$ represents the Hadamard product of two matrices.

THEOREM 4.1. Suppose that $A, H \in \mathbb{R}^{n \times n}$ and $D$ is a nonsingular diagonal matrix. Let $M=A \circ H$ and $N=M-A$. Then the spectrum of $M^{-1} N$ is unchanged if we replace $A$ with $D A D$.

Proof. Notice that $N=A \circ G$ where $G=e e^{T}-H$ and so $(D A D \circ H)^{-1}(D A D \circ G)=(D(A \circ H) D)^{-1} D(A \circ G) D=D^{-1}(A \circ H)^{-1}(A \circ G) D$. 
Similarity is not restricted to the splittings covered by this theorem, for example we can show that the result also holds for SSOR. To apply the result, we let $D=$ $\mathcal{D}\left(x_{k}\right)$ and look at the limit as $k \rightarrow \infty$. We see that

$\mathcal{D}\left(x_{k}\right) \mathcal{A}_{k} \mathcal{D}\left(x_{k}\right)=\mathcal{D}\left(x_{k}\right)\left(A+\mathcal{D}\left(x_{k}\right)^{-1} \mathcal{D}\left(A x_{k}\right)\right) \mathcal{D}\left(x_{k}\right) \rightarrow P+\mathcal{D}\left(x_{k}\right) \mathcal{D}\left(A x_{k}\right) \rightarrow P+I$,

where $P$ is doubly stochastic, and we can bound the asymptotic rate of convergence of our methods by looking at the spectrum of splitting matrices of $P+I$ (see Theorem 10.3.1 in [15]). In particular, in the case of Gauss-Seidel, we get a bound comparable with the formulation adopted in [13].

If $A$ is symmetric, then it is possible to significantly improve convergence speed over the SK algorithm with an appropriate choice of $M$. However, things are different if $A$ is nonsymmetric. For example, consider the effect of using Gauss-Seidel in (4.1) where $A$ is replaced by

$$
S=\left[\begin{array}{cc}
0 & A \\
A^{T} & 0
\end{array}\right]
$$

and $x_{k}=\left[\begin{array}{cc}r_{k}^{T} & c_{k}^{T}\end{array}\right]^{T}$. We have,

$$
\left[\begin{array}{c}
r_{k+1} \\
c_{k+1}
\end{array}\right]=\left[\begin{array}{c}
r_{k} \\
c_{k}
\end{array}\right]+\left[\begin{array}{cc}
\mathcal{D}\left(A c_{k}\right) \mathcal{D}\left(r_{k}\right)^{-1} & 0 \\
A^{T} & \mathcal{D}\left(A^{T} r_{k}\right) \mathcal{D}\left(c_{k}\right)^{-1}
\end{array}\right]^{-1}\left[\begin{array}{c}
\mathcal{D}\left(r_{k}\right)^{-1} e-A c_{k} \\
\mathcal{D}\left(c_{k}\right)^{-1} e-A^{T} r_{k}
\end{array}\right] .
$$

After some straightforward manipulation, this becomes

$$
\left[\begin{array}{c}
r_{k+1} \\
c_{k+1}
\end{array}\right]=\left[\begin{array}{c}
\mathcal{D}\left(A c_{k}\right)^{-1} e \\
\mathcal{D}\left(A^{T} r_{k}\right)^{-1} e+c_{k}-\mathcal{D}\left(c_{k}\right) \mathcal{D}\left(A^{T} r_{k}\right)^{-1} A^{T} r_{k+1}
\end{array}\right] .
$$

In the spirit of Gauss-Seidel, we can replace $r_{k}$ in the righthand side of (4.2) with $r_{k+1}$, giving

$$
\left[\begin{array}{c}
r_{k+1} \\
c_{k+1}
\end{array}\right]=\left[\begin{array}{c}
\mathcal{D}\left(A c_{k}\right)^{-1} e \\
\mathcal{D}\left(A^{T} r_{k+1}\right)^{-1} e
\end{array}\right]
$$

and this is precisely (2.2), the SK algorithm. In other words the Gauss-Seidel Newton method offers no improvement over the SK algorithm for nonsymmetric matrices.

There are any number of choices for $M$ in (4.1), but in tests we have not been able to gain a consistent and significant improvement over the SK algorithm when $A$ is nonsymmetric.

5. Conjugate gradient method. Recall that if $A$ is symmetric and nonnegative the Newton step (2.5) can be written

$$
\mathcal{A}_{k} x_{k+1}=A x_{k}+\mathcal{D}\left(x_{k}\right)^{-1} e,
$$


where $\mathcal{A}_{k}=A+\mathcal{D}\left(x_{k}\right)^{-1} \mathcal{D}\left(A x_{k}\right)$. By Theorems 3.1 and 3.5, we know that $\mathcal{A}_{k}$ is positive semi-definite and (2.5) is consistent whenever $x_{k}>0$ and we can solve the Newton step with the conjugate gradient method. Essentially, all we need to do is to find an approximate solution (2.5) and iterate, ensuring that we never let components of our iterates become negative. We now look in more detail at how we implement the method efficiently.

First, motivated by the proof of Theorem 3.1, we note that we can apply a diagonal scaling to (2.5) to give a symmetric diagonally dominant system. Premultiplying each side of the equation by $\mathcal{D}\left(x_{k}\right)$ and writing $y_{k+1}=\mathcal{D}\left(x_{k}\right)^{-1} x_{k+1}$ we get

$$
\left(B_{k}+\mathcal{D}\left(B_{k} e\right)\right) y_{k+1}=\left(B_{k}+I\right) e,
$$

where $B_{k}=\mathcal{D}\left(x_{k}\right) A \mathcal{D}\left(x_{k}\right)$. We needn't form $B_{k}$ explicitly, as all our calculations can be performed with $A$. The natural choice as initial iterate for every inner iteration ${ }^{4}$ is $e$, for which the initial residual is

$$
r=\left(B_{k}+I\right) e-\left(B_{k}+\mathcal{D}\left(B_{k} e\right)\right) e=e-B_{k} e=e-v_{k},
$$

where $v_{k}=x_{k} \circ A x_{k}$. Inside the conjugate gradient iteration we need to perform a matrix-vector product involving $B_{k}+\mathcal{D}\left(B_{k}\right)$. For a given vector $p$, we can perform this efficiently using the identity

$$
\left(B_{k}+\mathcal{D}\left(B_{k}\right)\right) p=x_{k} \circ\left(A\left(x_{k} \circ p\right)\right)+v_{k} \circ p .
$$

Since the rest of the conjugate gradient algorithm can be implemented in a standard way, this matrix-vector product is the dominant factor in the cost of the algorithm as a whole.

As a stopping criterion for the algorithm we use the residual measure

$$
\left\|e-\mathcal{D}\left(x_{k}\right) A x_{k}\right\|_{2}=\left\|e-v_{k}\right\|_{2} \text {. }
$$

In contrast to our experience with stationary iterative methods, it pays to run the inner iteration for a number of steps, and so we need a stopping criterion for the inner iteration, too. As is standard with inexact Newton methods, we don't need a precise solution while we are a long way from the solution and we adopt the approach outlined in [7, Chap. 3].

While we know that $B_{k} \mathcal{D}\left(B_{k}\right)$ is SPD if $x_{k}>0$, we cannot guarantee that (5.1) will have a positive solution. Furthermore, we do not know that our Newton method will converge if our initial guess is a long way from the solution. We therefore apply an Armijo-type rule [1] inside the inner iteration. We introduce a parameter $\delta$ which

\footnotetext{
${ }^{4}$ We also use $e$ as the default choice to start the algorithm.
} 
determines how much closer to the edge of the positive cone we are willing to let our current iterate move. By rewriting (2.5) in the form (5.1) we ensure that all coordinate directions are treated equally. Before we move along a search direction in the conjugate gradient algorithm, we check whether this would move our iterate closer to the edge than we are willing to go. If it does, we can either reject the step or only move part of the way along it and then restart the inner iteration. In our experience, it pays not to reject the step completely and instead we move along it until the minimum element of $y_{k+1}$ equals $\delta$. In general the choice $\delta=0.1$ seems to work well.

We report the results of our tests of the method in the next section. Our experience is that it is robust and can converge significantly quicker than the SinkhornKnopp algorithm.

If $A$ is nonsymmetric we can apply the same algorithm to

$$
S=\left[\begin{array}{cc}
0 & A \\
A^{T} & 0
\end{array}\right]
$$

From the results in $\S 3$ we know that the Jacobian will be singular at each step, but that the linear systems we solve will be consistent. As $B_{k}$ is singular, we cannot apply standard convergence theory. However our tests show our method is also robust in these cases, although the residual may not decrease monotonically, suggesting that the algorithm may be moving between local minima of the function $\mathcal{D}(x) S x-e$.

Rather than working with $S$, we can unravel the method and work directly with $A$ and $A^{T}$. This is particularly useful in solving the large scale balancing problems discussed in [9], where a rank one correction is applied implicitly to $A$.

As we mentioned early, although Khachiyan and Kalantari did not discuss the practical application of their algorithm in [8]. However it too can be implemented using the conjugate gradient method. For this formulation, (5.1) is replaced with

$$
\left(B_{k}+I\right) y_{k+1}=2 e .
$$

While this is straightforward to implement, we can no longer guarantee that our systems will be semi-definite unless $A$ is, too. In experiments, we have found that the Khachiyan-Kalantari approach is significantly slower than the one we propose.

6. Results. We now compared the performance of the conjugate gradient approach against a number of other algorithms. The algorithms considered are as follows. BNEWT is our implementation of an inexact Newton iteration with conjugate gradients; SK is the Sinkhorn-Knopp algorithm and EQ is the "equalize" algorithm from [16]; GS is a Gauss-Seidel implementation of (4.1). We use this in preference to the method outlined in [13], as for large matrices it is much easier to implement in MATLAB. In terms of rate of convergence, the algorithms are similar. 
We have tested the algorithm against both symmetric and nonsymmetric matrices, and also on matrices without total support. All our tests were carried out in MATLAB. We measure the cost in terms of the number of matrix-vector products taken to achieve the desired level of convergence. In our tests on nonsymmetric matrices, we have counted the number of products involving $A$ or $A^{T}$ (double the number for the symmetrised matrix $S$ ). We ran our algorithms until an appropriate residual norm fell below a certain tolerance. For the conjugate gradient algorithm, and other algorithms for symmetric matrices, we measured $\left\|\mathcal{D}\left(x_{k}\right) A x_{k}-e\right\|_{2}$ (replacing $A$ with $S$ if $A$ was nonsymmetric). For the Sinkhorn-Knopp algorithm we measured $\left\|\mathcal{D}\left(c_{k}\right) A^{T} r_{k}-e\right\|_{2}$, where $c_{k}$ and $r_{k}$ are defined in (2.2). In all cases, our initial iterate is a vector of ones and our default tolerance is $10^{-6}$.

In the conjugate gradient algorithm there are a number of parameters we can tune to improve performance connected to the convergence criterion of the inner step and the line search. Unless otherwise stated, we have just used the default values for these in an attempt to make our comparisons as fair as possible.

Our first test was on a selection of random sparse symmetric matrices, generated using the command $A=\operatorname{abs}(\operatorname{sprandsym}(n, a / n))+\operatorname{speye}(n) * .05$; for various values of $\mathrm{n}$ and $\mathrm{a}$. We added on a multiple of the identity matrix to ensure that our matrices were fully indecomposable. Our results are given in Table 6.1. The value of a is given in the first column, the value of $\mathrm{n}$ in the second row. Each entry represents the average cost for five different matrices: the cost varied considerably from matrix to matrix for both SK and GS, whereas BNEWT showed little variation. "Fail" represents an average cost of over 10000 matrix-vector products.

\begin{tabular}{|l|lll|lll|lll|}
\hline & \multicolumn{3}{|c|}{ BNEWT } & \multicolumn{3}{c|}{ SK } & \multicolumn{3}{c|}{ GS } \\
\hline & 100 & $10^{3}$ & $10^{4}$ & 100 & $10^{3}$ & $10^{4}$ & 100 & $10^{3}$ & $10^{4}$ \\
\hline 20 & 25 & 26 & 27 & 28 & 32 & 36 & 15 & 15 & 16 \\
10 & 31 & 33 & 36 & 47 & 69 & 261 & 21 & 26 & 29 \\
5 & 38 & 43 & 51 & 294 & 575 & 5195 & 35 & 56 & 72 \\
2 & 45 & 61 & 66 & 1588 & 8878 & Fail & 76 & 130 & 161 \\
1 & 47 & 56 & 70 & 1854 & Fail & Fail & 83 & 155 & 172 \\
\hline
\end{tabular}

The results show clearly the need to improve on SK as a balancing algorithm. The Gauss-Seidel approach works well in many examples, but eventually the superiority of BNEWT is evident. By tightening the tolerance condition we can improve the relative performance of BNEWT still further as it can take full advantage of super-linear convergence. 
Our next batch of test matrices were used by Parlett and Landis [16] to compare their balancing algorithms to the Sinkhorn-Knopp algorithm. These are all $10 \times 10$ upper Hessenberg matrices defined as follows. $H=\left(h_{i j}\right)$ where

$$
h_{i j}= \begin{cases}0, & j<i-1 \\ 1, & \text { otherwise }\end{cases}
$$

$H_{2}$ differs from $H$ in only that $h_{12}$ is replaced by 100 and $H_{3}=H+99 I$. Our results are given in Table 6.2. In this experiment, the tolerance changed to $10^{-5}$ as this was the choice in [16]. The cost for EQ is the ratio of SK operations to EQ operations, as reported in [16] (numbers less than one indicate SK is quicker.

\begin{tabular}{|l|l|l|l|l|}
\hline & BNEWT & SK & EQ & GS \\
\hline$H$ & 76 & 110 & 0.6 & 114 \\
$H_{2}$ & 90 & 144 & 0.8 & 150 \\
$H_{3}$ & 94 & 2008 & 8.9 & 2012 \\
\hline \multicolumn{4}{|c}{ TABLE 6.2 }
\end{tabular}

Rate of convergence for Parlett and Landis test matrices.

We see again the consistent performance of BNEWT, outperforming the other choices. The results for GS confirm our analysis in $\S 4$, showing it is virtually identical to SK.

We next tested BNEWT on the $n \times n$ version of $H_{3}$. For large values of $n$, this becomes very challenging to balance as the ratio between the smallest and largest elements of the balancing factors grows extremely large (the matrix becomes very close in a relative sense to a matrix without total support). The cost of convergence is given in Table 6.3, along with the ratio $r_{n}=\left(\max _{i} x_{i}\right) /\left(\min _{i} x_{i}\right)$.

\begin{tabular}{|l|l|l|l|l|}
\hline & $n=10$ & 25 & 50 & 100 \\
\hline BNEWT & 124 & 300 & 660 & 1792 \\
SK & 3070 & 16258 & 61458 & 235478 \\
$r_{n}$ & 217 & $7 \times 10^{6}$ & $2 \times 10^{14}$ & $2 \times 10^{29}$ \\
\hline
\end{tabular}

Rate of convergence for $H_{n}$.

BNEWT still copes in extremely trying circumstances, although the convergence of the residual is far from monotonic (we illustrate the progress after each inexact Newton step for $n=50$ in Figure 6.1. Recall that for nonsymmetric matrices, the Jacobian is singular and while our systems are consistent, it appears that the method is drawn towards local minima before finding an approximate solution. 


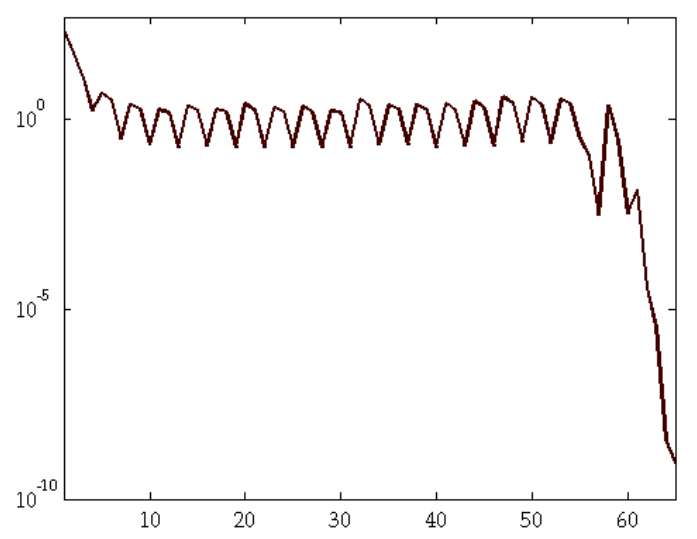

FIG. 6.1. Convergence graph of BNEWT for $\mathrm{H}_{50}$.

The oscillatory behaviour is undesirable, and it can be ameliorated somewhat by varying the parameters in BNEWT. This is illustrated in Figure $6.2\left(\eta_{\max }\right.$ is the maximum allowable tolerance for the inner iteration). In terms of cost, the choice $\eta_{\max }=10^{-2}, \delta=0.25$ proved best reducing the matrix-vector product count to 568 .

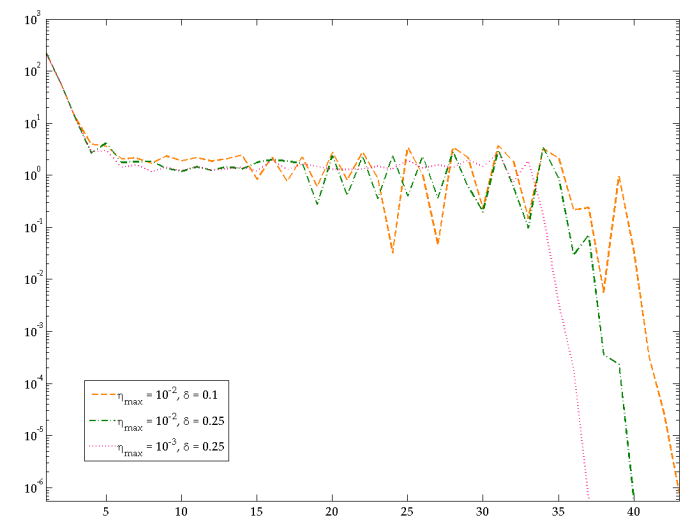

FIG. 6.2. Smoothing convergence of BNEWT for $\mathrm{H}_{50}$.

Finally we look at three matrices from the Harwell-Boeing collection [4], namely GRE185, GRE343 and GRE1107 (the number representing the dimension). The smallest of these was considered in [13] as a candidate for preconditioning, but convergence was slow. We illustrate the progress of BNEWT in Figure 6.3. The cost in matrix vector products (in ascending order of $n$ ) was 198, 112 and 320. Again, the method proves robust and we avoid the oscillatory behaviour we saw for our previ- 
ous more extreme example.

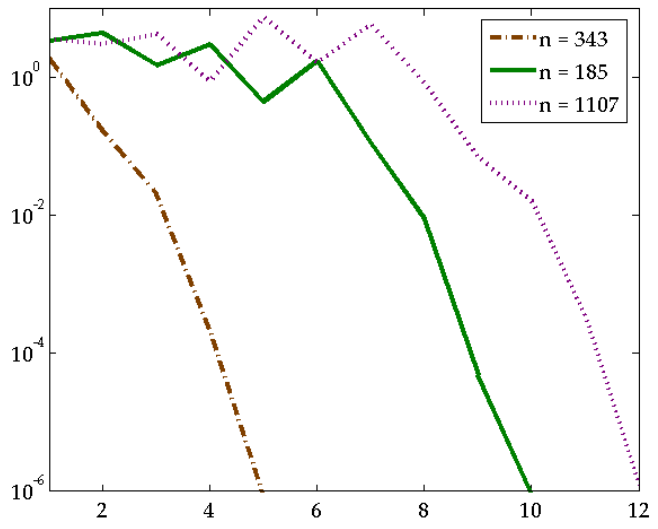

FIG. 6.3. Convergence of BNEWT for sparse nonsymmetric matrices.

\section{REFERENCES}

[1] L. ARMiJo, Minimization of functions having Lipschitz-continuous first partial derivatives, Pacific J. Maths., 16 (1996), pp. 1-3.

[2] Michael BaCharach, Biproportional Matrices \& Input-Output Change, CUP, 1970.

[3] Hamsa Balakrishnan, Inseok Hwang and Claire J. TOMLin, Polynomial approximation algorithms for belief matrix maintenance in identity management, Appears in Proceedings of the 43rd IEEE Conference on Decision and Control, Vol. 5 (2004), pp. 4874-4879.

[4] IAin S. Duff, R. G. Grimes And J. G. Lewis, Users' Guide for the Harwell-Boeing Sparse Matrix Collection (Release I), Technical Report RAL 92-086, Rutherford Appleton Laboratory (1992).

[5] MARTIN FÜRER, Quadratic convergence for scaling of matrices, in Proc. ALENEX/ANALC 2004, Lars Arge, Giuseppe F. Italiano, and Robert Sedgewick, editors, pp. 216-223, 2004.

[6] Bahman KalANTARI AND LEONID KHACHIYAN, On the complexity of nonnegative-matrix scaling, Linear Algebra Appl., 240 (1996), pp. 87-103.

[7] C. T. Kelley, Solving Nonlinear Equations with Newton's Method, SIAM, Philadelphia, 2003.

[8] LeONid KHACHIYAN AND BAHMAN KAlANTARI, Diagonal matrix scaling and linear programming, SIAM J. Opt., 2 (1992), pp. 668-672.

[9] Philip A. KNIGHT, The Sinkhorn-Knopp algorithm: convergence and applications, Technical Report 2006-8, Department of Mathematics, University of Strathclyde (2006).

[10] L. YU. Kolotilina, Nonsingularity/singularity criteria for nonstrictly block diagonally dominant matrices, Linear Algebra Appl., 359 (2003), pp. 133-159.

[11] B. LAmOND AND N. F. STEWART, Bregman's balancing method, Transportation Research 15B (1981), pp. 239-248.

[12] Nathan Linial, Alex SAmorodnitsky And AVi Wigderson, Deterministic Strongly Polynomial Algorithm for Matrix Scaling and Approximate Permanents, Combinatorica, 20 (2000) pp. 545-568.

[13] OREn E. Livne AND Gene H. Golub, Scaling by binormalization, Numerical Algorithms, 35 (2004), pp. 97-120. 
[14] Albert W. Marshall And Ingram OlKin, Scaling of matrices to achieve specified row and column sums, Numer. Math., 12 (1968), pp. 83-90.

[15] J. M. ORtega AND W. C. Rheinboldt, Iterative Solution of Nonlinear Equations in Several Variables, Academic Press, New York, 1970.

[16] B. N. PARLETT AND T. L. LANDIS Methods for scaling to doubly stochastic form, Linear Algebra Appl., 48 (1982), pp. 53-79.

[17] Micheal H. SChNeIder Matrix scaling, entropy minimization, and conjugate duality (II): The dual problem, Math. Prog. 48 (1990), pp. 103-124.

[18] RICHARD SINKHORN AND PAUL KNOPP Concerning nonnegative matrices and doubly stochastic matrices, Pacific J. Math. 21 (1967) , pp. 343-348.

[19] George W. Soules, The rate of convergence of Sinkhorn balancing, Linear Algebra Appl., 150 (1991), pp. 3-40.

[20] Olga TAUSSKY, Bounds for characteristic roots of matrices, Duke Math. J., 15(1948), pp. 1043-1044 


\section{Appendix. The Symmetric Algorithm.}

function $[\mathrm{x}, \mathrm{res}]=\operatorname{bnewt}(\mathrm{A}, \mathrm{tol}, \mathrm{x} 0, \operatorname{delta}, \mathrm{fl})$

$\%$ BNEWT A balancing algorithm for symmetric matrices

$\%$

$\% \mathrm{X}=\operatorname{BNEWT}(\mathrm{A})$ attempts to find a vector $\mathrm{X}$ such that

$\% \operatorname{diag}(\mathrm{X}) * \mathrm{~A} * \operatorname{diag}(\mathrm{X})$ is close to doubly stochastic. A must

$\%$ be symmetric and nonnegative.

$\%$

$\%$ XO : initial guess. TOL: error tolerance.

$\%$ DEL: how close balancing vectors can get to the edge of the

$\%$ positive cone. We use a relative measure on the size of elements.

$\% \mathrm{FL}$ : intermediate convergence statistics on/off.

$\%$ RES: residual error, measured by $\operatorname{norm}(\operatorname{diag}(\mathrm{x}) * A * \mathrm{x}-\mathrm{e})$.

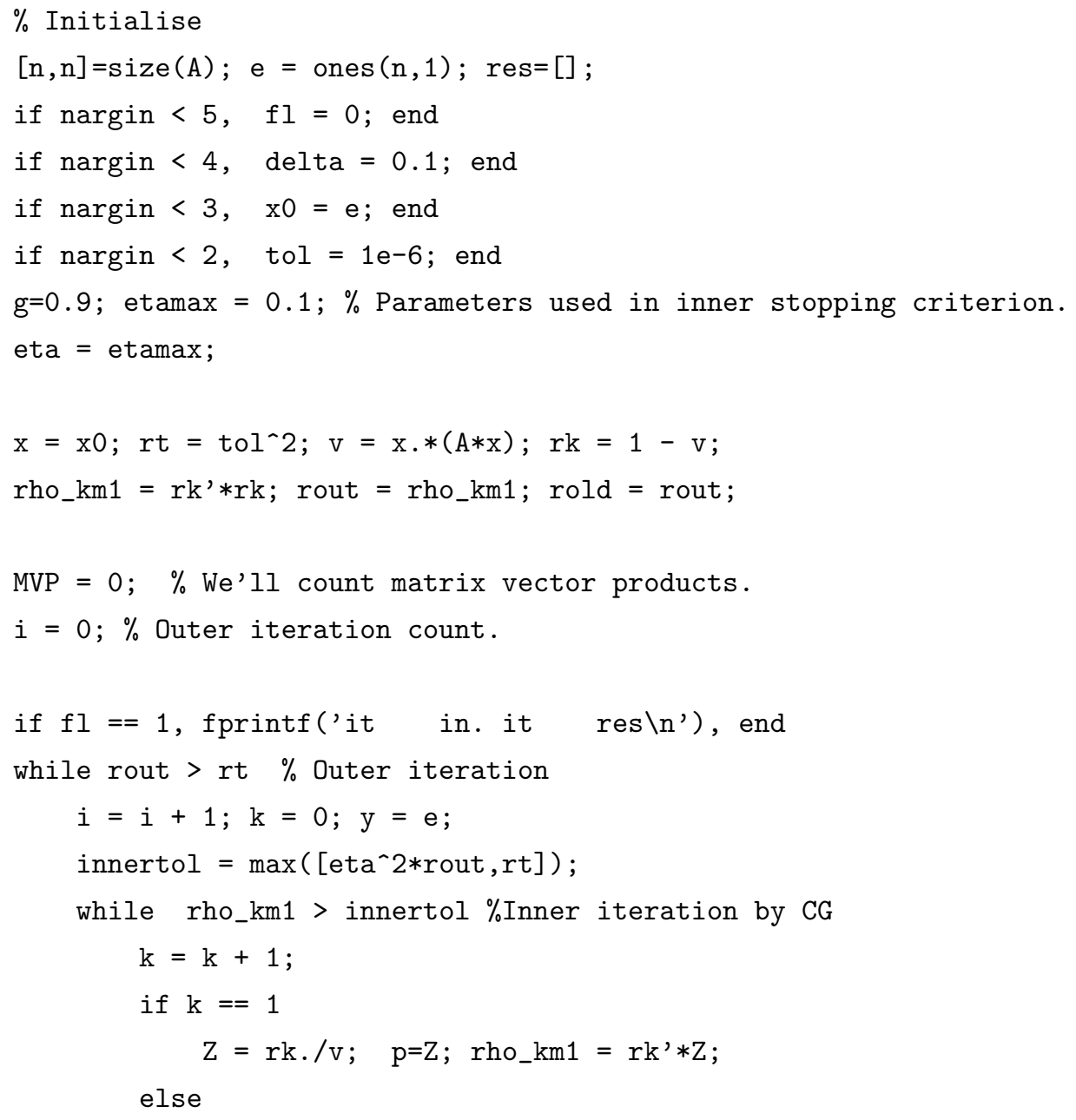




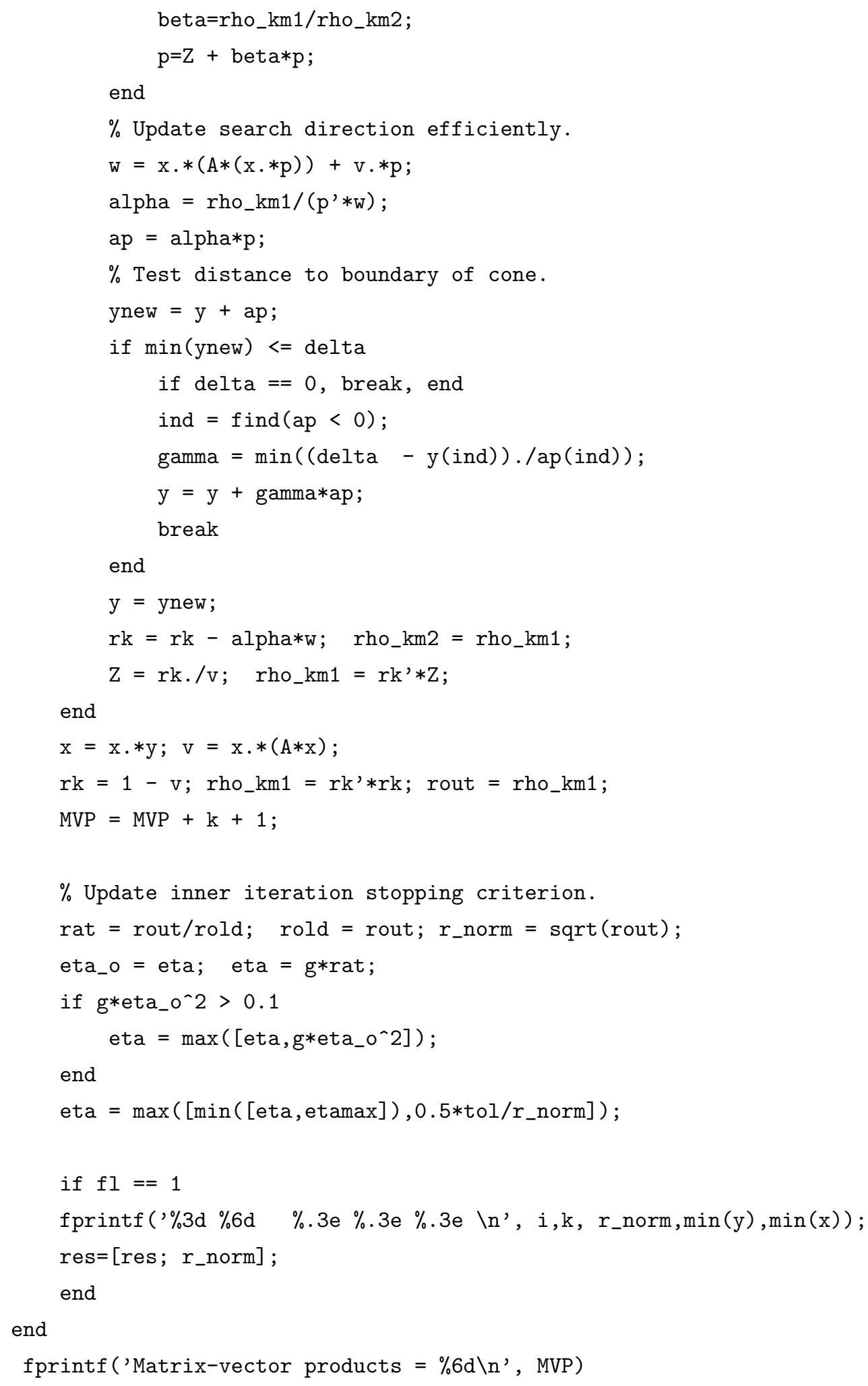

